\title{
Prophezeiung und Propaganda
}

\author{
Karl V., Pamphilus Gengenbach und die Erwartung \\ eines zweiten Kaisers Karl im Jahr 1519
}

Von ERwin Frauenknecht

Das Landesarchiv Baden-Württemberg verwahrt im Hauptstaatsarchiv Stuttgart an unerwarteter Stelle ein bislang unbekanntes Exemplar eines Einblattdrucks mit einer Prophezeiung auf einen Kaiser Karl ${ }^{1}$. Der Druck bietet den Text der Prophezeiung mit einem Portrait Karls V. Die Weissagung kündigt einen Herrscher Karl an, den Sohn Philipps, der nach einer Reihe von erfolgreichen Kriegen viele Völker unterwerfen werde und mit einer zweifachen Krone gekrönt werde. Er werde fast über die gesamte Erde herrschen, dann nach Jerusalem auf den Ölberg ziehen, dort seine Krone niederlegen und im 35. Jahr seiner Herrschaft sterben. Vom Engel gekrönt, werde er als Vater der Erde der erste Kaiser nach Friedrich III. sein. Die Titelzeile des Druckes gibt einen Hinweis auf die Vorlage; nach diesen Angaben stamme der Text aus einem alten Codex eines berühmten Doktor Cobole und sei von ihm selbst 1440 geschrieben worden:

Fuit inventum in quodam codice antiquissimo famosissimi doctoris Cobole, qui fuit scriptus propria eius manu, Anno.1440. pro ut sequitur. Qui doctor obiit Anno .1480 .

Carolus Hispaniarum rex et Austriae archidux.

Carolus Philippi filius longam habens frontem, alta supercilia, oculos grossos, nasum aquilinum, etatis suae quartodecimo coronabitur, vel secundum aliam scripturam Anno septimodecimo. Magnum congregabit exercitum. Et omnes tyrannos regni sui destruet. Nam sicut sponsus et sponsa, ita erit sociata cum eo iusticia. Vsque ad vicesimumquartum annum sui regni, adducet bella, subiugans Anglicos, Hispanos, Arragonos et Gallos, Longobardos, Romam cum Florentia subingans destruet et igne comburet, duplicem coronam obtinebit. Et postmodum mare transiens cum maximo exercitu lustrabit Greciam et Grecorum rex nominabitur. Chaldeos, Turcos, Palestinos atque Georgianos subiugabit, faciens edictum, quod quicumque non adoraverit signum crucis, morte moriatur, et non erit qui ei posit resistere. Fere

${ }^{1}$ Landesarchiv Baden-Württemberg, Abt. Hauptstaatsarchiv Stuttgart A 80 Bü 4. 
universae terrae dominabitur. His factis, veniet ad sanctam Hierusalem, ascendens montem Oliveti, deponensque de capite coronam, deo gratias agens, cum signis et miraculis emittet spiritum, Anno sui regni tricesimoquinto. Hic coronatus erit ab angelo, Pater terrae, et primis imperator post Fridericum tertium.

Von diesem Druck ist bisher lediglich ein weiteres, schlechter erhaltenes Exemplar bekannt, das heute in der Bibliothèque Nationale in Paris aufbewahrt wird. Denise Hillard hat es vor einiger Zeit in einem kleinen Beitrag vorgestellt, identifiziert und in seinen historischen Kontext eingeordnet ${ }^{2}$. Der Druck stammt aus der Offizin des Basler Druckers und Dichters Pamphilus Gengenbach ${ }^{3}$, wie schon die beiden Initialen am Ende des Textes nahe legen. Die Zuordnung des Einblattdruckes hat Kerstin Prietzel in ihrer umfassenden Studie zum buchdruckerischen Werk Gengenbachs bestätigt und präzisiert ${ }^{4}$. Der Einblattdruck wurde im Vorfeld der Wahl Karls V. zum römisch-deutschen König 1519 in Basel gedruckt. Das Blatt ist nach Prietzel Teil einer umfangreichen verlegerischen Initiative Gengenbachs und gehört zu einer Reihe von Texten, mit der der Basler Drucker seiner Habsburgerverehrung im Allgemeinen und seine Parteinahme für Karl V. im Speziellen Ausdruck verleihen wollte. Gengenbach stellt sich mit dieser „zweite(n) Produktionswelle aktueller historischer Literatur“ aus seiner Offizin in den Dienst der habsburgerfreundlichen Partei in $\mathrm{Basel}^{5}$. Zu diesem politischen Interesse kommt hinzu, dass Gengenbach bereits in früheren Texten das Sujet der prognostischen Literatur verarbeitete. Sein berühmtes Fastnachtsspiel „Der Nollhart“, 1516 verfasst und 1517 in Basel aufgeführt, rezipierte unter anderem bereits eine Endkaiser-Weissagung in der Überlieferungstradition des sogenannten Pseudo-Metho$\operatorname{dios}^{6}$.

Der hier vorzustellende neue Fund bietet Anlass für Beobachtungen in drei Richtungen. Zum einen ist zu fragen nach dem Überlieferungskontext des Stuttgarter Exemplars, zum anderen lassen sich zur Bildvorlage Gengenbachs neue Erkenntnisse gewinnen und drittens schließlich wirft die Herkunftsangabe zu Beginn der Prophetie Fragen nach der Verbreitung dieses prognostischen Textes auf.

2 Denise Hillard, Charles Quint: Élection et prophetie, in: Bulletin du bibliophile 1 (1993) S. 7-22.

3 Vgl. Kerstin Prietzel, Artikel „Gengenbach, Pamphilus“, in: Deutscher Humanismus 1480-1520. Verfasserlexikon, hg. von Franz Josef Worst вrocк, Bd. 1, Berlin 2008, Sp. 889904.

${ }^{4}$ Kerstin Prietzel, Pamphilus Gengenbach, Drucker zu Basel (um 1480-1525), in: Archiv für Geschichte des Buchwesens 52 (1999) S. 229-461. In der Druckchronologie wird der Druck unter der Nr. 52, S. 281 unter dem Titel „Dr. Cobola: Fuit inventum ..." beschrieben.

${ }^{5}$ Vgl. Kerstin Prietzel, Kaiserlob im eidgenössischen Basel, in: Literatur - Geschichte - Literaturgeschichte. Beiträge zur mediävistischen Literaturwissenschaft. Festschrift für Volker Honemann zum 60. Geburtstag, hg. von Nine Miedema/Rudolf Suntrup, Frankfurt am Main 2003, S. 569-584, Zitat hier S. 576.

${ }^{6}$ Hannes Möhring, Der Weltkaiser der Endzeit. Entstehung, Wandel und Wirkung einer tausendjährigen Weissagung, Stuttgart 2000, S.291-317, hier S. 340. 


\section{Der Überlieferungskontext des Stuttgarter Einblattdruckes}

Zum archivalischen Befund: Ungewöhnlich ist der heutige Aufbewahrungsort des Einblattdruckes; er wird unter der Signatur A 80 Bü 4 heute im Landesarchiv Baden-Württemberg, Abteilung Hauptstaatsarchiv Stuttgart, aufbewahrt ${ }^{7}$. Vor seiner 2012 erfolgten Neuordnung befand sich der Druck im gleichen Bestand in Büschel 1, dort als Nummer 9 eingeordnet. Schon seit dem 16. Jahrhundert ist dieser Bestand als Archivmembrum nachgewiesen, war jedoch größeren Umstrukturierungen unterworfen ${ }^{8}$. Die Spuren dieser Extraditionen und Umschichtungen sind im maßgeblichen Repertorium, das von Wilhelm Ferdinand Ludwig Scheffer (1779-1826) zu Beginn des 19. Jahrhunderts angefertigt wurde, heute noch sichtbar. Scheffer, der von 1791 bis 1826 Geheimer Archivar war, verzeichnet den Einblattdruck, Büschel 1 zugehörig, unter der fortlaufenden Nummer 9: Imago Caroli Hispaniae Regis inventa in quodam antiquissimo Codice famosissime Doctoris Cobole ao. 1440. Ein späterer Zusatz vermerkt dazu im Repertorium: Carolus V ${ }^{9}$.

Etwas Licht in die Provenienzgeschichte lässt sich bringen, wenn man ältere Findbücher zu Rate zieht. Die katastrophalen Verwüstungen nach der Schlacht bei Nördlingen 1634 hatten sowohl das herzogliche Archiv als auch die Bibliothek stark in Mitleidenschaft gezogen ${ }^{10}$. Nach der Rückkehr Herzog Eberhards III. im Jahr 1638 versuchten die beiden bestellten Archivare Johann Konrad Heller und Johann Jakob Hornmold ab 1639 Ordnung in die geretteten Bestände zu bringen. In einer dieser Übersichten findet sich der früheste Nachweis, dass sich der vorgestellte Einblattdruck im heutigen Bestand A 80 befand. Johann Konrad Heller (1595-1661) hat diese Zusammenstellung zwischen 1640 und 1651 verfertigt. Ähnlich wie in dem späteren Findbuch von Scheffer zitiert der fragliche Eintrag den Titel des Einblattdruckes ${ }^{11}$. Dagegen fehlt der Druck in der älteren Übersicht von David Förter $(† 1631)$ aus dem Jahr 1617. Aus diesem Vergleich darf man schließen, dass der Druck nach 1617 und vor 1640 in den jetzigen Überlieferungszusammen-

7 Landesarchiv Baden-Württemberg, Abt. Hauptstaatsarchiv Stuttgart A 80: Online-Findbuch, bearbeitet von Andrea Неск; permalink: http://www.landesarchiv-bw.de/ plink/?f=1-1374346 (letzter Zugriff 12.01.2014).

${ }^{8}$ Vgl. dazu Hans-Martin Maurer/Stephan Molitor/Peter Rückert (Bearb.), Übersicht über die Bestände des Hauptstaatsarchivs Stuttgart. Altwürttembergisches Archiv (A-Bestände) (Veröffentlichungen der Staatlichen Archivverwaltung Baden-Württemberg, Bd.32), Stuttgart ${ }^{2} 1999$, S. 58. - Dieses Büschel enthält etwa auch eine Überlieferung der Goldenen Bulle von 1356, vgl. dazu jetzt Erwin Frauen knecht, Die Goldene Bulle und ihr Druck von Lienhart Holl in Ulm 1484, in: ZWLG 70 (2011) S. 127-138.

9 HStA Stuttgart A 80 Bü 1: „Beziehungen zu den deutschen Kaisern, Korrespondenzen, kaiserliche Mandate“.

10 Vgl. dazu Eugen Schneider, Zur Geschichte des württembergischen Staatsarchivs, in: WVjH NF 12 (1903) S. 3-22, hier S. 9 ff.

${ }^{11}$ HStA Stuttgart A 605 Bü II 80, Faszikel 2, Blatt 2r; wie im späteren Repertorium von Scheffer ist der Druck als Nr. 9 mit seinem Titel verzeichnet: „Imago Caroli Hispaniae Regis inventa in quoddam ..." 
hang gekommen sein muss. Von 1611 bis 1662 waren das herzogliche Archiv und die Hofbibliothek in Stuttgart organisatorisch nicht getrennt und bis zum 18. Jahrhundert verwahrte das Archiv auch nachweislich Bücher oder andere Kunstgegenstände ${ }^{12}$. Dieser Sachverhalt kann erklären helfen, wieso sich der Einblattdruck in diesem ungewöhnlichen Überlieferungszusammenhang befindet.

\section{Zur bildlichen Darstellung: Das Portrait Karls V.}

Das Portrait des Einblattdruckes zeigt Karl im Profil als jungen Herrscher mit kinnlangen Haaren und Barett (Abb. 1). Die Barettkrempe ist geschlitzt und wird von einem Tuch durchzogen. Auf den Schultern trägt Karl einen Pelzkragen, darauf liegt die Ordenskette des Ordens vom Goldenen Vlies. Das Portrait ist situiert vor einer Landschaft und eingerahmt durch zwei Baumstämme, die jeweils ein Wappen tragen.

Einen Datierungsansatz gibt die Titulatur vor: Carolvs Hispaniarum rex et Austriae archidux. Der spanische Königstitel und die österreichische Erzherzogswürde werden angesprochen, nicht dagegen der Titel als römisch-deutscher König. Die Titulatur passt daher nur in den historischen Kontext zwischen der Erhebung Karls zum spanischen Thronfolger 1516 und der Königswahl im Reich am 28. Juni 1519.

Woher bezog der Drucker Pamphilus Gengenbach sein Bild Karls V.? Die Frage ist von einigem Interesse, zumal das verwendete Abbild Karls Merkmale eines Portraits im Sinne einer individuellen Erkennbarkeit beanspruchen kann. Die frühe deutsche Portraitkunst hat sich erst um 1500 als eigenes Genre herausgebildet, auch von diesem Ansatzpunkt her erhält die Frage nach der Bildvorlage ihre Berechtigung $^{13}$. Zu kurz greift meiner Meinung nach die Bewertung des Portraits bei Prietzel: „Im Zentrum steht der Portraitholzschnitt Karls V., der zwar hübsch anzuschauen ist, aber keinerlei inhaltliche Aspekte abdeckt.“" ${ }^{14}$ Inhaltliche Merkmale transportieren bereits die beiden über dem Portrait angebrachten Wappen. Für illiterate Betrachter boten sie eine Identifikationsmöglichkeit, denn dargestellt sind das Habsburgerwappen und das burgundische Wappen. Anhand dieser Wappen war eine Zuschreibung der dargestellten Person möglich.

\footnotetext{
12 Vgl. dazu Michael KLein (Bearb.), Die Handschriften der Sammlung J 1 im Hauptstaatsarchiv Stuttgart (Die Handschriften der Staatsarchive in Baden-Württemberg, Bd.1), Wiesbaden 1980, S.20f.

${ }^{13}$ Vgl. dazu den Ausstellungskatalog Das Unsichtbare sichtbar machen. Deutsche Portraits um 1500, in: Dürer - Cranach - Holbein. Die Entdeckung des Menschen: Das deutsche Portrait um 1500, Katalog des Kunsthistorischen Museums Wien und der Kunsthalle der Hypo-Kulturstiftung München, hg. von Sabine Haag u.a., München 2011.

${ }^{14}$ Prietzel, Pamphilus (wie Anm. 4) S. $331 \mathrm{f}$.
} 
Vollends deutlich aber wird der Stellenwert des Portraits, wenn man dazu die unmittelbar verwendete Vorlage in Betracht zieht. An Untersuchungen zur Rolle des höfischen Portraits im Umfeld Karls V. mangelt es nicht. Nach Karl Schütz stellt sich die zeitliche Verteilung der Bilder so dar, dass vor allem aus der frühen Jugendzeit Karls zahlreiche Portraits angefertigt wurden, während für die Phasen, in der sich Karl in Spanien aufhielt, kaum Abbildungen erhalten sind ${ }^{15}$. Gerade im Vorfeld der Wahl 1519 bestand dann die Notwendigkeit, Karl im Reich bekannt zu machen, dazu bediente man sich auch der Druckgrafik, wie Rainer Wohlfeil herausgearbeitet hat ${ }^{16}$. Mehrere dieser graphischen Darstellungen entstanden auf Initiative habsburgischer Parteigänger, zum einen im Umfeld der Statthalterin Margarete und zum anderen vor allem in Augsburg.

Bisher nicht beachtet wurde, dass insbesondere eine Radierung aus der Werkstatt des Augsburger Eisenätzers Daniel Hopfer ${ }^{17}$ eine auffallende Ähnlichkeit mit der Darstellung Karls V. im vorgestellten Einblattdruck zeigt (Abb. 2). Hopfers Radierung weist in der Physiognomie des Gesichts und in der Ausstattung die gleichen Merkmale auf: die gehöckerte Nase Karls, die kinnlangen Haare und die zeitgenössische höfische Bekleidung mit Barett und Pelzkragen, auf dem ebenfalls die Ordenskette liegt. Eine Umschrift in Versalien enthält Name und Titulatur des Dargestellten: Karolvs REX CATOLICVS ${ }^{18}$. Nach Metzger stellt Hopfers Radierung das „früheste Bildnis Karls als König von Spanien“ dar und dürfte „um oder kurz nach 1516 “ entstanden sein ${ }^{19}$. Bis in die Details stimmt diese Vorlage mit dem Bild Karls auf Gengenbachs Einblattdruck überein, verändert wurde allerdings die

15 Karl Sснӥтz, Karl V. und die Entstehung des höfischen Protraits, in: Kaiser Karl V. (1500-1558). Macht und Ohnmacht Europas, hg. von Wilfried SeipeL, Wien 2000, S. 57-63, besonders S. 58. - Zur umfangreichen jüngeren Literatur über Karl V. vgl. vor allem Luise SCHORN-SchütTE, „In seinem Reich ging die Sonne nicht unter“. Neue Literatur zu Kaiser Karl V. (1500-1558), in: HZ 277 (2003) S. 379-387.

16 Rainer Wohlfeil, Grafische Bildnisse Karls V. im Dienste von Darstellung und Propaganda, in: Karl V. 1500-1558. Neue Perspektiven seiner Herrschaft in Europa und Übersee, hg. von Alfred Kohler/Barbara Haider/Christine OtTner (Zentraleuropastudien, Bd. 6), Wien 2002, S. 21-56, besonders S. $28 \mathrm{ff}$.

$17 \mathrm{Vgl}$. den umfangreichen Bestandskatalog von Christof Metzger, Daniel Hopfer. Ein Augsburger Meister der Renaissance. Eisenradierungen, Holzschnitte, Zeichnungen, Waffenätzungen. Katalog zur gleichnamigen Ausstellung der Staatlichen Graphischen Sammlung München in der Pinakothek der Moderne, München 5. November 2009 bis 31. Januar 2010, München 2009. - Zur Biographie vgl. auch den Beitrag von Tobias Günther, Handwerk und Kunst an der Schwelle zur Neuzeit. Der Kaufbeurer Radierer Daniel Hopfer (um 1470 bis 1536), in: Das Rätsel von St. Martin. Archäologische Argumente, Indizien und Hypothesen zur Frühgeschichte der Stadt von Kaufbeuren, hg. von Stadtarchiv und Heimatverein Kaufbeuren e.V. (Kaufbeurer Schriftenreihe, Bd. 4), Thalhofen 2002, S. 50-79.

$18 \mathrm{Vgl}$. Metzger (wie Anm. 17) Abb. 94, S. 205; vgl. auch Wohlfeil (wie Anm. 16) Abb. 1.

19 Metzger (wie Anm. 17) S. 420. Hopfers Vorlage wiederum hat nach Metzger eine Bildnismedaille des Gian Maria Pomedello abgegeben, die als Andenken an die spanische Königsproklamation entstanden war. 


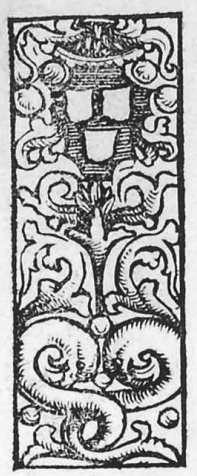

\section{E-Gíntintentuntinquodar cor dice antiquiffimo famofiffimi doctoris Cobole, qui fus} it fcriptus propria eius manu, Anno.1440.pro ut fequitur. Qui dector obijt Anno.1480

CAROLVS HISPANIARVM $R E X$

\section{A. R C H I D V X.}
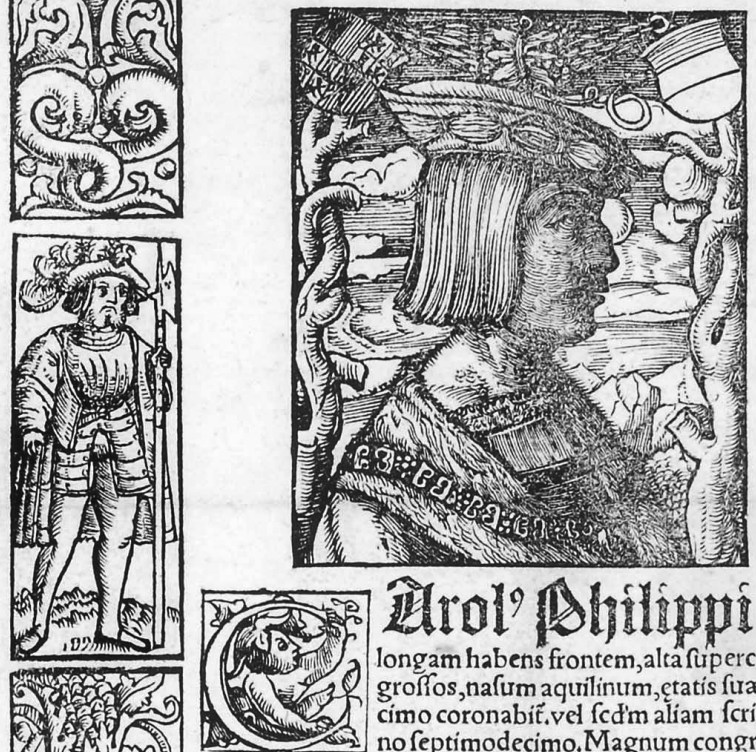

(1)
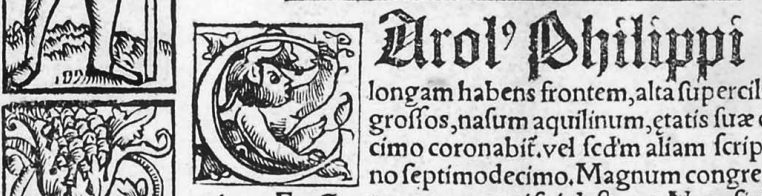

longm habens frontem, of grofios, nafum aquilinum, tatis furæ quartode cimo coronabiêt.vel fcđ'm aliam fcripturã Ans nofeptimodecimo.Magnum congregabitex/ ercitum. Et om̃es tyrannos regnifui deftruet. Nam ficut fpöfus \& fponfa, ita erítfociata cum eo iufticia, $V$ føg ad vicefimữquar tum annum fui regni, adducet bella, fubiugans Anglicos, His fpanos, Arragoros \& Gallos, Longobardos, Romã cum Flo. rentia fubiugans deftruet $\mathcal{Q}$ igne cóburet, duplicem coronam obtinebit. Et poftmodữ mare tranfiens cưm maximo exercitu luftrabit Greciam, \& Gręcorum rex nominabitur. Chaldeos Turcos, Paleftinos atç Georgianos fubiurgabit, faciens ediçũu, q quicứç non a dorauerit fignum crucis, morte moriatur, \& nô erit qui ei poffit refiftere, Fere vniuerfæterræ dominabitur. His factis, veniet ad fanctam Hierufalem, afcendens montem Oliue ti, deponenfog de capite coronam, deo gratias a gens, curm fignis 8 miracPis emittet fpiritfi, Anno fui regni tricefimoquinto. Hic coronatuserit $a b$ angelo, Pater terr $x_{3}$ et primus imperator polt Fridericum tertium,

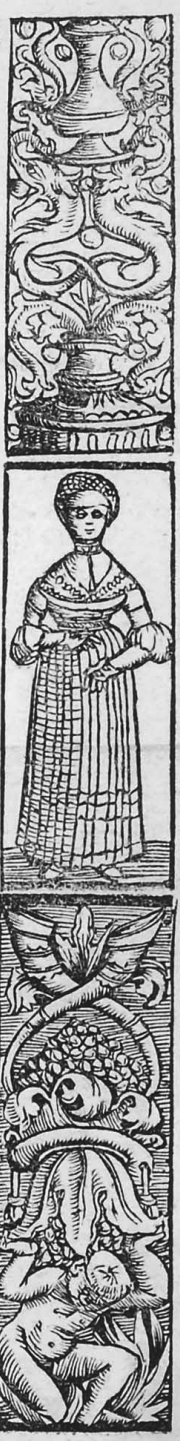

Abb. 1: Prophetie auf Karl V. Druck aus der Basler Offizin des Pamphilus Gengenbach, 1519 (Vorlage: HStA Stuttgart A 80 Bü 4). 
Abb. 2: Profilbildnis Karls V. Eisenradierung von Daniel Hopfer, um 1516/1519 (Vorlage: Germanisches Nationalmuseum Nürnberg, Graphische Sammlung, Inventar-Nr. K 10028, Kapsel-Nr. 127).

[Die Abbildung kann aus rechtlichen Gründen online nicht bereitgestellt werden.] 
Titulatur, indem der Ehrentitel Rex catolicus durch die oben genannten Bezeichnungen Carolvs Hispaniarum rex und Austriae archidux ersetzt wurde.

Hopfers Radierung muss in großer Stückzahl hergestellt worden sein. Zumindest deuten die Abnutzungserscheinungen der Platte auf eine enorme Auflagenhöhe der Radierung hin ${ }^{20}$. Das wiederum korrespondiert mit der Intention des Bildes, weil für die bevorstehende Wahl das Bild Karls möglichst breit publik gemacht werden sollte. Es ist daher nahe liegend, dass Gengenbach das enorm weit verbreitete Bild aus der Augsburger Werkstatt Hopfers zur Verfügung hatte und in seinem Flugblatt mit der Prophezeiung kombinierte. Gengenbachs eigene schöpferische Leistung besteht in der Verknüpfung der prognostischen Texttradition mit dem druckgrafischem Portrait Karls V. Bisher kursierte die verwendete Prophezeiung ohne Bildkomponente, ikonographische Ergänzungen kommen in der Überlieferungstradition der Prophezeiung äußerst selten vor, schon gar nicht in der konkreten Verbindung, in die sie Gengenbach gestellt hat ${ }^{21}$.

Vielleicht war Gengenbachs Zugriff auf seine Portraitvorlage noch direkter, denn der Basler Drucker hatte unmittelbare Kontakte nach Augsburg. In seiner Offizin war in dieser Zeit der aus Augsburg stammende Ambrosius Holbein ( $\dagger$ nach 1519) als Holzschneider tätig ${ }^{22}$. Zusammen mit seinem jüngeren Bruder Hans Holbein der Jüngere hielt er sich spätestens seit 1516 in Basel auf, künstlerisch im Schatten seines berühmteren Bruders ${ }^{23}$. Ambrosius Holbein fertigte 1517 etwa den Titelholzschnitt für Gengenbachs Ausgabe der Lobrede des Mailänder Humanisten und Sekretärs Karls V., Luigi Marliani (†1521), die dieser auf dem 18. Kapitel des Ordens vom Goldenen Vlies gehalten hatte ${ }^{24}$. Der Holzschnitt zeigt das Wappen des spanischen Königs, Karls berühmtes Motto Quy vouldra sowie die Ordenskette, mithin Elemente, die auch das Titelbild des Einblattdruckes aufnimmt. Desweiteren benutzte Gengenbach zwischen 1518 und 1524 Zierinitialen, die Ambrosius Holbein hergestellt hatte ${ }^{25}$.

An der Gestaltung des Portraits selbst war Holbein nach Ausweis der kunsthistorischen Forschung nicht unmittelbar beteiligt - es wird von Franz Hieronymus dem unbekannten Meister GZ zugeschrieben, der ebenfalls für Gengenbach als Holzschneider tätig war ${ }^{26}$. Das Portrait wurde eigens für die Propagandaoffensive

${ }^{20}$ Ebd., S. 420.

${ }^{21}$ Eine Herrscherdarstellung findet sich in einer Überlieferung der Karlsprophetie aus dem 15. Jahrhundert in einer Handschrift der Bayerischen Staatsbibliothek München, vgl. dazu Leonie von Wilckens, Die Prophetien über die Päpste in deutschen Handschriften, in: Wiener Jahrbuch für Kunstgeschichte 28 (1975) Abb. 205.

${ }^{22}$ Hillakd (wie Anm. 2) S. 11.

${ }^{23}$ Vgl. dazu Christian Müller/Stephan Kemperdick (Hg.), Hans Holbein der Jüngere. Die Jahre in Basel 1515-1532, München 2010.

24 Prietzel, Kaiserlob (wie Anm. 5) S. 576.

25 Prietzel, Pamphilus (wie Anm. 4) S. 264.

26 Vgl. Franz Hieronymus, Oberrheinische Buchillustration, Bd. 2: Basler Buchillustration, 1500-1545, Ausstellungskatalog, Basel 1984; Hillard (wie Anm. 2) S. 10. 
Gengenbachs hergestellt und fand in mehreren Drucken aus seiner Offizin um 1520 Verwendung. Nach der Druckchronologie, die Prietzel in ihrer umfassenden Untersuchung zur Druckerei Gengenbachs ermitteln konnte, fand das Portrait erstmals im vorliegenden Einblattdruck Verwendung. Eine weitere Verwendung ist fassbar in einem Preislied auf die Wahl Karls, wohl unmittelbar auf die Wahl im Juni 1519 auch in Gengenbachs Offizin entstanden, und auch das im Frühjahr 1520 entstandene sogenannte Wiener Prognosticon, das der Basler Drucker an den neugewählten Habsburger richtete, verwendet das Bild Karls ebenso. Schließlich trägt auch das 1521 bei Gengenbach erschienene und Johann Eberlin von Günzburg zugeschrieben Werk der „Fünfzehn Bundesgenossen“ das Portrait Karls V. ${ }^{27}$

\section{Zum Text: Die Verbreitung der Prophetie}

Die Prophezeiung selbst ist unter dem modernen Titel „The Second Charlemagne“ (Reeves) in der Literatur über Endkaiser-Weissagungen sehr wohl präsent und gehört in eine Texttradition, die im späten Mittelalter in prognostischen Variationen von der Erwartung eines zweiten Kaisers mit Namen Karl ausging. Art und Umfang dieser Weissagungen waren unterschiedlich, gemeinsam war allen die Vorstellung, dass am Ende der Zeit der letzte Kaiser ein Franzose mit Namen Karl sein werde ${ }^{28}$. Die Urfassung der Prophezeiung ist bald nach der Krönung des französischen Königs Karl VI. im Jahr 1380 in Frankreich fassbar. Der Text prophezeit, dass „Karl, der Sohn eines Karl aus der hochberühmten Nation der Lilie“ gekrönt werde sowie Ordnung und Gerechtigkeit wieder herstellen werde ${ }^{29}$.

Wenig später taucht die Prophezeiung bereits im Umkreis weiterer joachimitischer Weissagungen auf. In einer Handschrift der Vaticana etwa wird der Text gleich im Anschluss an den bekannten Libellus des Telesphorus von Cosenza angefügt. Diese Version überliefert, neben kleineren Textveränderungen, vor allem einen erweiterten Schluss mit dem am Ende angefügten Hinweis, Karl werde von einem angelico pastore gekrönt werden und nach dem gegenwärtigen Schisma der erste Kaiser nach dem dritten Friedrich sein: Hic coronatus erit ab Angelico pastore

27 Vgl. Prietzel, Pamphilus (wie Anm. 4) Nr. 52, 53, 57 und Nr. 70. Auch die Ornamentik und figurale Gestaltung der Randleisten (Landsknecht) findet ihre Parallelen in zeitgleichen Druckerzeugnissen Gengenbachs.

${ }^{28}$ Vgl. dazu Marjorie Reeves, The influence of prophecy in the later middle ages. A Study in Joachimism, Oxford 1969, besonders S.320ff.; Hannes MöHring, Der Weltkaiser der Endzeit. Entstehung, Wandel und Wirkung einer tausendjährigen Weissagung, Stuttgart 2000, hier S. 291-317 mit weiterer Literatur. Zur Verwendung im Umfeld Karls V. vgl. die allgemeinen Hinweise bei Ferdinand Sегвт, Karl V. Der Kaiser und die Reformation, Berlin 1990, S. $20 \mathrm{ff}$.

${ }^{29}$ Maurice Chaume, Une prophetie relative a Charles VI., in: Revue du moyen age latin 3 (1947) S. 27-42, hier Edition S. 29; Möhring (wie Anm. 28) S.298; Reeves (wie Anm.28) S. 328 . 
et primus Imperator post Federicum tercium, post presens scisma et tribulationes et persecutiones pseudoprophetarum et dicti Federici ${ }^{30}$. Dieser Schluss verknüpft eine weitere, populäre mittelalterliche Weissagung, nämlich die von einem engelsgleichen Papst (pastor angelicus), unter dessen Pontifikat sich am Ende der Zeiten eine Reform der Kirche und die Ausbreitung des Christentums über alle Menschen vollziehen werde ${ }^{31}$.

Über Karl VI. (†1422) hinaus wurde die Prophezeiung in Frankreich auf nachfolgende Herrscher mit dem Namen Karl übertragen. Der Text wurde zur bedeutendsten politischen Weissagung des fünfzehnten und sechzehnten Jahrhunderts ${ }^{32}$. In gelehrten Kreisen war er nicht unbekannt, der Historiograph Thomas Ebendorfer $(† 1464)$ zitiert ihn etwa am Ende des sechsten Buches seiner Chronik der römischen Könige, die Ebendorfer nach 1449 auf ausdrücklichen Wunsch des Habsburgers verfasst hatte ${ }^{33}$. Verbreitung fand die Prophetie aber vor allem durch ihre Aufnahme in verschiedene, zum Teil anonyme Sammelhandschriften mit anderen prophetischen Texten ${ }^{34}$.

Besonders die militärischen Erfolge Karls VIII. über die neapolitanische Flotte 1494 und der sich anschließende Zug über Florenz und Rom nach Neapel führten in Frankreich und Italien dazu, in Karl VIII. den prophezeiten zweiten Kaiser Karl zu sehen ${ }^{35}$. Literarische Kreise in Frankreich griffen die Prophezeiung auf, übersetzten die lateinischen Version ins Französische und münzten sie auf Karl VIII. $\mathrm{um}^{36}$. Auch in Italien kursierte die Weissagung und wurde ebenfalls auf Karl VIII. bezogen. Nach dem Einzug Karls in Florenz im November 1494 spielte Savonarola auf die Prophezeiung eines zweiten Karls an und kaprizierte diese Hoffnungen auf den französischen König ${ }^{37}$. Das Vatizinium blieb, aktualisiert auf Karl VIII., im $\mathrm{Umlauf}^{38}$.

Ganz neue Aktualität floss der Prophezeiung zu, als nach dem Tod Maximilians 1519 die Wahl des römisch-deutschen Königs anstand. Die Prophezeiung wurde nun auf den Habsburger Karl V. bezogen. Karl vereinte Vieles: Als Enkel Maximilians und dessen Frau Maria von Burgund ließ sich eine Verbindung zu den franzö-

30 Aus der Handschrift Vat. Reg. lat. 580 fol. 52r ediert bei Reeves (wie Anm. 28) S. 328.

31 Möhring (wie Anm. 28) S. $269 \mathrm{ff}$.

32 Reeves (wie Anm. 28) S. 330.

33 Thomas Ebendorfer, Chronica regum Romanorum, hg. von Harald Zimmermann, Teil 1 (MGH Scriptores rerum germanicarum, N.S. 18), Hannover 2003, S. 625 f. - Vgl. dazu demnächst Franz Fuchs/Wilfried Hartmann: Friedrich III. - der dritte Friedrich der Kaiserprophetien?; an dieser Stelle sei Herrn Prof. Franz Fuchs (Würzburg) ganz herzlich für die Überlassung des Manuskripts gedankt.

34 Eine Zusammenstellung bei Reeves (wie Anm. 28) Appendix B, Nr. 23, S. $531 \mathrm{ff}$.

35 Möhring (wie Anm. 28) S. 299.

36 Vgl. Chaume (wie Anm. 29) S. 32 f.; vgl. Möhring (wie Anm. 28) S. 300.

37 Möhring (wie Anm. 28) S. 301.

38 Reeves (wie Anm. 28) S. 330 f. stellt eine Reihe von Handschriften des 15. und 16. Jahrhunderts vor, die zum überwiegenden Teil aus Italien stammen und in denen die Prophezeiung überliefert wird. 
sischen Königen herstellen. Die Prophezeiung auf einen „französischen“ Endkaiser konnte nun auch in Deutschland aufgegriffen werden, allerdings war dazu eine Textmodifikation nötig. In der ursprünglichen Version war der Endkaiser Karl Sohn eines gleichnamigen Kaisers Karl. In der jüngeren Version lautete der Anfang: Carolus Philippi filius ${ }^{39}$. Wann diese Version entstand, muss offen bleiben ${ }^{40}$.

In dieser personalisierten Form taucht der Text wiederholt auch schon weit im Vorfeld der Wahl Karls am 28. Juni 1519 auf und fand eine enorme Beachtung. Nach dem Urteil von Reeves darf er sogar als die beliebteste politische Prophetie des 15. und 16. Jahrhunderts gelten ${ }^{41}$.

Die genauen Überlieferungs- und Vermittlungswege bleiben vielfach im Dunkeln. Ein Beispiel dafür bietet Göbel Schickenberges, Vogt des westfälischen Augustinerchorherrenstifts Böddeken bei Paderborn. Er hinterließ Aufzeichnungen, zumeist Amts- oder Rechnungsbücher, in die er chronikalische Notizen und Reiseaufzeichnungen eingetragen hatte. Nach eigenen Angaben notierte Schickenberges die lateinische Form der Kaiserprophetie bereits am 13. April 1519, also noch vor der Wahl im Juni, ohne allerdings seine Quelle anzugeben ${ }^{42}$. Das von Schickenberges mitgeteilte Kolophon der Prophezeiung erklärt dazu: Repertum est Verone in antiquossimo (!) et autentico libro. Unklar ist, woher der westfälische Abt seine Textversion bezog, der Herausgeber Rüthing beklagt zu Recht die „defizitäre Erforschung dieser Prophetie ${ }^{\text {“43 }}$, schränkt aber seine Schlussfolgerungen zu sehr ein: „Die Prophetie soll 1519 in Venedig gedruckt worden sein; doch ob Göbel bereits am 13. April dieses Jahres ein Exemplar zur Verfügung stand, ist fraglich“. Man muss nicht zwingend eine gedruckte Fassung als Vorlage annehmen, es kann auch eine handschriftliche Überlieferung in Frage kommen, die Schickenberges benutzt hatte. Schon Friedrich von Bezold wies auf eine solche Texttradition der Prophetie hin, die angeblich im Jahr 1505 „zu Verona in einem uralten Buch“ entdeckt worden $\operatorname{sei}^{44}$. Allgemein ist festzuhalten, dass eine ganze Reihe von Samm-

39 Möhring (wie Anm. 28) S.304f.; zum weiteren Kontext vgl. auch Reeves (wie Anm. 28) S. 350 und Georg R. Spohn, Eine deutsche Karl-Prophezeiung von 1519 in einem kurpfälzischen Kopialbuch, in: Archiv für Kulturgeschichte 52 (1970) S. 226-243, hier S. 239.

40 Vgl. dazu Möhring (wie Anm. 28) S. 305, der es als nicht zwingend ansieht, das Jahr 1500 (das Geburtsjahr Karls V.) als terminus post quem anzunehmen, weil auch ein Bezug der Prophetie auf Karl den Kühnen, den Sohn Philipps des Guten, nicht ausgeschlossen werden kann.

${ }^{41}$ Reeves (wie Anm. 28) S. 330 und ebd., Appendix B, Nr. 23, S. 531 mit einer Zusammenstellung der Überlieferungen.

${ }^{42}$ Die Chronik Bruder Göbels. Aufzeichnungen eines Laienbruders aus dem Kloster Böddeken 1502 bis 1543, hg. von Heinrich RüThing (Quellen und Forschungen zur Kirchen- und Religionsgeschichte 7), Bielefeld 22006, S.155f.; vgl. dazu auch Martin Hille, Providentia Dei, Reich und Kirche. Weltbild und Stimmungsprofil altgläubiger Chronisten 1517-1618 (Schriftenreihe der Historischen Kommission bei der Bayerischen Akademie der Wissenschaften, Bd. 81), Göttingen 2010, S. 259.

${ }^{43}$ Ebd., S. 155, Anm. 22.

${ }^{44}$ Friedrich von Bezold, Zur deutschen Kaisersage, in: Sitzungsberichte der königlich 
lungen prophetischer Texte kursierte, deren Zusammenhänge im Einzelnen noch nicht zufriedenstellend aufgearbeitet sind.

Auf die verschiedenen Formen der Herkunftsangaben in den anonymen Prophezeiungen, die den Überlieferungszusammenhang und die Autorschaft mehr verschleiern als erklären, ist Roberto Rusconi näher eingegangen. Nach seinen Feststellungen kursierten solche Prophetien in Italien Ende des 15. und Anfang des 16. Jahrhunderts von Hand zu Hand und gerade die älteren prognostischen Texte wurden mit scheinbar prägnanten Herkunftsangaben („in quodam antiquo libello“) versehen, um die Authentizität des Textes zu erhöhen ${ }^{45}$.

Vor diesem Hintergrund gilt es, die Zuschreibung unseres Einblattdruckes in den Blick zu nehmen, denn die Prophetie wird folgendermaßen angekündigt: Fuit inventum in quodam codice antiquissimo famosissimi doctoris Cobole, qui fuit scriptus propria eius manu anno 1440, pro ut sequitur. Qui doctor obiit anno 1480.

Auch hier wird auf das hohe Alter der Vorlage hingewiesen, zudem noch mit einer konkreten Autorangabe. Ein sehr berühmter Doktor Cobola habe den Text 1440 mit eigener Hand geschrieben; dieser Cobola sei zudem 1480 gestorben. Bisher ist es nicht gelungen, mit diesen Lebensdaten eine historische Person zu identifizieren.

Ottavia Niccoli hat vor einiger Zeit die Zirkulation der prognostischen Texte im Italien des 15. Jahrhunderts in den Blick genommen und dabei auf den Maler und Historiographen Leone Cobelli aus Forli hingewiesen. In seinem Besitz befand sich ein „libriciolo de [...] profecie“, das er 1480 aus franziskanischen Kreisen bekommen hatte ${ }^{46}$. Auch die Lebensdaten von Cobelli ${ }^{47}$ passen nicht zu den Angaben unseres Textes, zudem lässt sich nicht erkennen, welchen Inhalt das heute verlorene Büchlein hatte. Aber das Beispiel ist meiner Meinung nach geeignet, um zu zeigen, wo die Provenienz des von Gengenbach verwendeten und mit dem Bild Karls V.

bayerischen Akademie der Wissenschaften, Philosophisch-philologische Klasse 1884, München 1885, S.600. - Siehe auch Roberto Rusconi, „Ex quodam antiquissimo libello“. La tradizione manoscritta delle profezie nell'Italia tardomedioevale: dalle collezioni profetiche alle prime edizioni a stampa, in: The use and abuse of eschatology in the middle ages, hg. von Werner Verbene/Daniel Verhelst/Andries Welkenhuysen (Mediaevalia Lovaniensia, Bd.15), Leuven 1988, S.440-471, besonders S.452. - Die Herkunftsangabe "aus Verona“ taucht schließlich auch auf in einer weiteren deutschen Fassung, die 1532 anonym in dem Traktat „Erzelung der Kunigreich in Hispanien ... mer eine alte prophecy Kayser Carl betreffend" (VD 16 E 3913).

${ }^{45} \mathrm{Vgl}$. Roberto Rusconi, Les collections prophétiques en Italie à la fin du moyen âge et au début des temps modernes. Remarques à propos de divers manuscrits italiens conservés dans les bibliothèques de Paris, in: Mélanges de L'Ecole française de Rome 102 (1990) S. 481511, hier S. 483 f.; vgl. dazu auch Reeves (wie Anm. 28) S. 330 f. und Ottavia Niccoli, Profeti e popolo nell'Italia del rinascimento, Rom 1987, S. 22 f. zu zirkulierenden Sammlungen und ebd., S. 221 ff. zur vorliegenden Prophetie.

${ }^{46}$ Niccoli (wie Anm. 45) S. 25.

$47 \mathrm{Zu}$ Leone Cobelli vgl. E. Menestò, Leone Cobelli, in: Dizinario biografico degli Italiani, Bd. 26 (1982), S. 431-433 mit weiterer Literatur. 
verknüpften Textes vermutet werden dürfte - in Italien. Es wird also nicht hinreichend klar, woher der Drucker Gengenbach die Textvorlage für seinen Druck bezog, mögliche Spuren können nur angedeutet werden.

Wenn der Text der Prophezeiung in der zugrunde liegende Version um $1500 \mathrm{zu-}$ nächst in Italien verbreitet war, so scheint eine Vermittlung über Humanistenkreise nicht unwahrscheinlich. Allerdings fand Gengenbach in den humanistischen Briefwechseln der Zeit keine Erwähnung - eine Ausnahme, wenn man bedenkt, wie häufig fast alle übrigen Basler Drucker, man denke nur an Amerbach oder Froben, in humanistischen Briefen Erwähnung fanden ${ }^{48}$.

Dennoch lassen sich zumindest im Spätsommer 1518 Indizien für eine Annäherung an den Basler Humanistenkreis feststellen, wohl vermittelt über Wolfgang Capito. Der Druck zweier Luthertexte sowie eine insgesamt stärkere Ausrichtung der Offizin in den Jahren 1517 und 1518 auf lateinische Texte, die stärker auf ein gebildetes Publikum ausgerichtet waren, weisen in diese Richtung ${ }^{49}$. Doch können auch die diffusen Kontakte zum Basler Humanistenkreis die Herkunft der Prophezeiung nicht weiter erhellen.

Es sei noch eine zweite Spur angeführt. Im Juni 1517 druckte Gengenbach die Oratio in comitiis ordinis Aurei velleris ${ }^{50}$ des Luigi Marliani, ein Text, der Marlianis Rede auf dem 18. Kapitel des Ordens vom Goldenen Vlies aus dem Jahr 1516 widergibt und eine Lobrede auf Karl darstellt. Die graphische Ausstattung des Textes weist, wie oben erwähnt, mit dem Wappen und dem Motto Karls bereits Elemente auf, die im Holzschnitt der Prophetie wieder aufgegriffen werden. In einer Art Fürstenspiegel liefert Marliani Vorschläge für eine gute Regierung und streicht für Karls Herrschaft günstige Vorzeichen heraus. Der Mailänder Humanist Marliani (†1521) gehörte als Leibarzt, dann als Sekretär zum engsten Umfeld Karls in Spanien und hatte vor allem in den Jahren 1516/1517 großen Einfluss auf den jungen spanischen Herrscher ${ }^{51}$.

Wie der unmittelbare Kontakt zwischen Marliani, dem Berater Karls, und Gengenbach, dem Basler Drucker zustande kam, ist unklar. Der Druck zeigt aber, dass Gengenbach schon einige Jahre vor der eigentlichen Publikationsoffensive zugunsten Karls V. 1519 Sympathien für die Position der Habsburger zeigte ${ }^{52}$.

Und über den italienischen Humanisten Luigi Marliani wäre zudem auch eine direkte Vermittlung weiterer Texte zugunsten Karls denkbar. Doch dazu fehlen bisher weitere Belege.

Die eben diskutierte Zuschreibung der Prophetie an einen Doktor Cobola kann aber helfen, eine weitere Überlieferung des Textes einzuordnen. Georg R. Spohn ${ }^{53}$

\footnotetext{
48 Prietzel, Pamphilus (wie Anm. 4) S. 330.

49 Ebd., S. 329.

50 Ebd., Nr. 31, S. 275.

51 Ebd., S. 328.

52 Ebd.

53 Spohn (wie Anm. 39).
} 
hatte vor längerer Zeit eine deutsche Version der Prophezeiung vorgestellt, die als Abschrift im Kopialbuch des Kurfürsten Ludwig V. (1478-1544) von der Pfalz 1519 eingetragen wurde. Spohn versuchte - ohne Kenntnis unseres Einblattdruckes diese deutsche Version in einen historischen Kontext zu setzen und stellte weitreichende Überlegungen zum Autor an. Es könne sein, so Spohn, dass „ein Doktor Zobel, gestorben 1480, tatsächlich im Jahre 1440 ... eine lateinische oder vielleicht schon deutsche Fassung der Karl-Prophezeiung in ein Buch eingetragen hat, die nach 1500 von einem Unbekannten [...] redigiert und 1519 in der kurpfälzischen Kanzlei kopiert wurde ${ }^{454}$. Spohn versuchte dann weiter, einen Doktor Zobel im pfälzischen Raum dingfest zu machen, listet auch eine Reihe von Namen auf, gestand aber gleichzeitig ein, dass keine der aufgeführten Personen mit dem in der Prophetie genannten Cobola identisch sein könne ${ }^{55}$.

Mit der Kenntnis des vorliegenden Einblattdrucks aus Basel lassen sich zumindest Spohns Überlegungen zur Einordnung des Textes aus der pfälzischen Kanzlei präzisieren. Die unmittelbare Vorlage für die deutsche Übersetzung der Prophetie im Kopialbuch Ludwigs V. hat ohne Zweifel Gengenbachs Druck abgegeben. Darauf deutet schon die wortgleich übersetzte Zuschreibung hin, mit der das Vaticinium im pfalzgräflichen Kopialbuch angekündigt wird: Nachfolgend Prognostication ist gefunden worden in einem alten buch wylandt doctor zobels welichs geschrieben ist worden mit siner aignen handt 1440 der vorgemelt doctor ist gestorben 1480 jar. ${ }^{56}$ Nicht mit Sicherheit zu entscheiden ist dagegen, ob die deutsche Übersetzung der Prophetie auch in der kurpfälzischen Kanzlei hergestellt wurde und ein Exemplar von Gengenbachs Druck somit auch in Heidelberg vorhanden war. Beide Textüberlieferungen, sowohl Gengenbachs Druck als auch die auf ihn zurückgehende deutsche Übersetzung, bilden aber zumindest einen eigenen Strang in der vielgestaltigen Überlieferungssituation der Prophetie auf Karl V.

Nach Spohns Feststellungen wurde die Übersetzung im Kopialbuch Ludwigs V. von der Pfalz im unmittelbaren Umfeld zu weiteren Texten zur Wahl Karls V. platziert. Vorausgehen im Kopialbuch weitere Aufzeichnungen zur Wahl im Juni 1519, und direkt nach der Prophetie folgt eine Abschrift des Dankesschreibens des Pfälzer Kurfürsten an den neugewählten Karl V. Daraus lässt sich schließen, dass der Text Mitte Juni auf jeden Fall schon in der Heidelberger Kanzlei bekannt sein musste.

Dass man in Heidelberg in diesen Jahren äußerst interessiert an den Vorgängen im Reich war, steht außer Frage, und das mag die Kenntnis des Textes in der pfälzischen Kanzlei erklären. Die anstehende Nachfolgeregelung im Reich bot für die nach dem Landshuter Erbfolgekrieg politisch und territorial angeschlagene Pfalz

54 Ebd., S. 241. Auch die Überlegungen zur Datierung dieser Version, die Spohn S. 241 anstellt, werden in der Forschung skeptisch beurteilt, vgl. dazu MöHring (wie Anm.28) S. 305 mit Anm. 149.

55 Spohn (wie Anm. 39) S. 242.

56 Ebd., S. 226. 
neuen Handlungsspielraum, insofern wird man in Heidelberg alle Aktivitäten um die anstehende Wahl interessiert registriert haben.

Der pfälzische Kurfürst Ludwig tendierte zunächst aus taktischen Gründen zur Unterstützung der französischen Kandidatur Franz I., wechselte allerdings 1518 in das Lager der Unterstützer Karls V. Es war kein Geheimnis, dass der Parteiwechsel durch Kaiser Maximilian mit der Anerkennung der Pfalz als Reichslehen sowie weiterer verbriefter Privilegien honoriert wurde. Auch beträchtliche Geldzuwendungen haben den Pfälzer Kurfürsten in das Lager der Wähler Karls V. gelockt ${ }^{57}$. Die Platzierung des prognostischen Textes im Umfeld anderer relevanter Texte zur Wahl im Frühsommer 1519 erscheint daher nicht überraschend.

Zurück zu unserem Text. Eine genaue Untersuchung der Textgenese des prognostischen Werkes, die hier nicht geleistet werden kann, müsste auch den Schlusssatz einbeziehen: Die Krönung durch einen Engelspapst (bic coronatus ab angelico pastore), wie oben dargelegt ein Bezug auf eine gängige joachimitische Weissagung, die in allen älteren Versionen der Prophetie durchweg enthalten ist, ist im vorliegenden Einblattdruck und entsprechend in der deutschen Übersetzung aufgegeben: Hic coronatus erit ab angelo, Pater terrae, et primus imperator post Fridericum tertium. Auch hier ist der Druck Gengenbachs der erste Textzeuge, der im Schlusssatz auf den Engelspapst verzichtet. In der prognostischen Literatur des späten Mittelalters galt der engelsgleiche Papst am Ende der Zeiten als Garant einer Kirchenreform und als Pendant zur Vorstellung eines Endkaisers; deren Verzicht darf also durchaus als klares Bekenntnis für Karl V. gelten. Sprachlich wirkt die Formulierung etwas holprig, auch in der deutschen Übersetzung wirkt das nach (der wurdt gekronet von den Engeln und ein vatter des Ertreichs) ${ }^{58}$, aber in ähnlicher Form wird der Schlusssatz auch in der einflussreichen Reformschrift Onus ecclesiae des Chiemseer Bischofs Berthold Pürstinger geboten: hic ab angelo coronabitur tanquam patrie pater. Nach eigener Angabe habe Pürstinger die Prophetie 1519 erhalten und zwar aus einer 1505 in Italien erschienenen Version; diesen Text hat der Chiemseer Bischof dann 1524 in sein anonym gedrucktes Werk aufgenommen ${ }^{59}$.

Abseits dieser sprachlichen Details zeigt die Prophezeiung, dass sie im Umfeld der Wahl Karls V. durchaus breite Beachtung fand. Gengenbachs Verdienst ist es, der Prophetie durch den Druck in seiner Verbindung mit einem Portrait des zu wählenden Königs Karl V. neuen Schwung verliehen zu haben. Die Version, die Pamphilus Gengenbach in seinem Einblattdruck bietet, repräsentiert einen eigenen Überlieferungszweig der populären Weissagung. Seine Rezeption fand der Druck auch in einer deutschen Übersetzung, die kurz nach der Wahl 1519 in einem pfalzgräflichen Kopialbuch eingetragen wurde.

57 Vgl. dazu zusammenfassend Meinrad Sснанв, Geschichte der Kurpfalz, Band 2: Neuzeit. Stuttgart/Berlin/Köln 1992, S.15f.

58 Spohn (wie Anm.39) S. 227.

59 Möhring (wie Anm. 28) S. 305. 
Die Aufnahme des Textes in das pfälzische Kopialbuch verdeutlicht auch, dass die Prophetie in politischen Kreisen sehr wohl zur Kenntnis genommen wurde. Der Vorgang ist vielleicht in Parallele zu setzen mit der Rezeption des Textes in Venedig. Auch hier ist die Prophetie bald nach der Wahl Karls nachzuweisen. Der außerordentlich produktive venezianische Schriftsteller und Historiograph Marin Sanudo (1466-1536) berichtet in seinem Hauptwerk, den Diarii, fast täglich von 1496 bis zum September 1533 über politische und gesellschaftliche Ereignisse in der Lagunenstadt. Ende November 1519 rückt er eine lateinische Version der Prophetie in seine Aufzeichnungen ein, mit dem Hinweis: Prophetia missa Venetiis ex Anglia anno Domini. ${ }^{60}$ Die genaue Herkunft von Sanudos Version bleibt allerdings bislang ebenso im Dunkeln, wie die Quelle, aus der Pamphilus Gengenbach seinen Text bezog.

60 Diarii, hg. von Rinaldo Fuli u.a., Bd. 28, Venedig 1890, Sp. 132 f. - In der älteren Literatur fälschlich als englische Übersetzung Sanudos angeführt, bei MöHRING (wie Anm. 28) S. 305 die Richtigstellung; zur Person Sanudos vgl. Christiane NeER feld, „Historia per forma di Diarii“. Venezianische Gegenwartschronistik um 1500, Diss. Rom, 2001, S. 27-43. 